\title{
Influencia de las condiciones del tiempo y almacenamiento en la estabilidad dimensional de los moldes fabricados a partir de hidrocoloides irreversibles
}

\section{Influence of the conditions of time and storageon dimensional stability of casts made from Irreversible hydrocolloid}

\section{Resumen}

Objetivo: Evaluar los cambios dimensionales de los modelos de yeso a partir de impresiones con hidrocoloide irreversible (alginato) bajo distintos tiempos de vaciado y condiciones de almacenamiento. Métodos: Estudio experimental in vitro que realizó 130 impresiones con hidrocoloide irreversible (Tropicalgin ${ }^{\circledR}$ ) sobre un modelo maestro. Las impresiones fueron asignadas en 13 grupos dependiendo del tiempo de vaciado (a los 5, 10 y 15 minutos), según las condiciones de almacenamiento (uso de torundas de algodón y/o empaques herméticas) y combinando las condiciones con los tiempos de vaciado. Las medidas de los modelos en yeso (altura y diámetro) fueron comparadas con las mediciones del modelo maestro para calcular los cambios dimensionales. Resultados: A nivel de la altura se obtuvo un cambio dimensional de $0,3 \pm 0,22 \mathrm{~mm}$ con el uso de torundas, sellado hermético y vaciado a los 15 minutos (décimo tercer grupo), mientras que con el vaciado inmediato (primer grupo) se obtuvo un cambio de $0,41 \pm 0,38 \mathrm{~mm}(p=0,03)$. A nivel del diámetro el cambio dimensional del décimo tercer grupo fue de 0,08 $\pm 0,12$ $\mathrm{mm}$ mientras que con el primer grupo fue de $0,06 \pm 0,04 \mathrm{~mm}(p=0,41)$ siendo estos los grupos con mayores cambios dimensionales. Conclusiones: Los grupos de modelos con impresiones almacenadas con torundas y empaques herméticos evidenciaron los menores cambios dimensionales a los 5, 10 y 15 minutos de tiempo de vaciado. Los modelos obtenidos con los tiempos de vaciado a los 10, 15 minutos y sin condiciones de almacenamiento demostraron los mayores cambios dimensionales tanto en altura como en diámetro.

Palabras clave: Impresión; Materiales de impresión dental; Precisión de la medición dimensional; Técnica de impresión dental.
ISSN: $1560-9111$

Artículo Original

Carlos Roca-Sacramento 1,a, Luisa lbarra-Vásquez 1,a, Juan Diego Amado-Chavez 1,a, Alexis Saucedo-García ${ }^{1, a}$, Yuri Castro-Rodríguez 2,b

${ }^{1}$ Sociedad Científica de Estudiantes de Odontología. Universidad Nacional Mayor de San Marcos. Lima, Perú.

${ }^{2}$ Universidad Nacional Mayor de San Marcos,

Facultad de Odontología. Lima, Perú.

a Estudiante del pregrado.

${ }^{\mathrm{b}}$ Cirujano Dentista.

Correspondencia:

Carlos Roca-Sacramento

Correo electrónico: carlos_tkd94@hotmail.com Universidad Nacional Mayor de San Marcos,

Facultad de Odontología. Calle Germán Amézaga 375. Lima 1, Perú.

\section{Coautores:}

Luisa Ibarra-Vásquez

luisa.ibarra@unmsm.edu.pe

Juan Diego Amado-Chavez

juan.amado@unmsm.edu.pe

Alexis Saucedo-García

alexis.saucedog@unmsm.edu.pe

Yuri Castro Rodríguez

yuricastro_16@hotmail.com

Conflicto de intereses: Los autores declaran no tener conflictos de interés.

Fuente de financiamiento: Autofinanciado

Fecha de recepción: 15/09/17

Fecha de aceptación: 27/03/18 
while with immediate emptying (first group) a change of $0.41 \pm 0.38 \mathrm{~mm}(p=0.03)$, being these the groups with mostdimensional changes. Regarding the diameter level, the dimensional change of the thirteenth group was $0.08 \pm 0.12 \mathrm{~mm}$; while the first group was $0.06 \pm 0.04 \mathrm{~mm}(p=0.41)$. Conclusions: The groups of models with impressions stored with cotton rolls and hermetic packs showed the smallest dimensional changes at 5,10 and 15 minutes of emptying time. The models obtained with the emptying times at 10,15 minutes and without storage conditions showed the greatest dimensional changes in both height and diameter.

Keywords: Dental impression materials; Dental impression technique; Dimensional measurement accuracy; Printing.

\section{Introducción}

Los registros de impresión dental son procedimientos que deben ser realizados de forma adecuada que permitan la obtención de modelos definitivos óptimos, ya sean para el diagnóstico y/o tratamiento.

De los distintos materiales de impresión, los más utilizados son los hidrocoloides irreversibles (alginatos) principalmente por su facilidad en el manejo y el bajo costo que suponen ${ }^{1,2} ;$ sin embargo, la reproducción positiva (modelo de yeso) puede alterarse debido a que el alginato durante su gelificación puede sufrir cambios dimensionales que se ven influenciados por el tiempo de vaciado, condiciones de almacenamiento, uso de agentes desinfectantes y variaciones en las dosis utilizadas ${ }^{3}$. Los cambios dimensionales de los hidrocoloides irreversibles pueden ocurrir por dos fenómenos: imbibición (ganancia de agua por mantener la impresión en un ambiente húmedo) y sinéresis (pérdida de agua por exposición prolongada de la impresión al ambiente), procesos que dependen de factores externos como la temperatura del ambiente, humedad y almacenaje por periodos prolongados 4,5 . Se conoce, por ejemplo, que a mayor tiempo transcurrido desde la toma de impresión se generan mayores cambios dimensionales ${ }^{6}$.

Pese al avance en el desarrollo de los elastómeros, los alginatos destacan por su sencilla manipulación y múltiples usos ${ }^{7}$. La reproducción de detalles finos de los hidrocoloides es aproximadamente $25 \%$ menor, por lo que estos materiales no son utilizados en impresiones donde se requiera una alta fidelidad del detalle, como en impresiones para incrustaciones, coronas, puentes fijos, carillas ${ }^{8}$. Su uso se limita a las zonas con poca retención debido a que son propensos a los desgarres.

Diversas modificaciones de los alginatos han permitido mejorar sus propiedades, de esta forma, las presentaciones cromáticas ofrecen mayor confort al odontólogo, además de ofrecer una mejor reproducción de las estructuras a impresionar ${ }^{5}$. Algunos autores ${ }^{1,9,10}$ han indicado que los cambios dimensionales durante el proceso de gelificación son diferentes según marca comercial y que en el caso de los cromáticos se facilita la identificación de los tiempos de gelificación. Durante las actividades clínicas del odontólogo se suele seleccionar a los alginatos como principales materiales de impresión y que por limitaciones físicas y de logística, en muchas ocasiones, realizar el vaciado inmediato de la impresión se dificulta y/o es postergada con lo que se corre el riesgo de modificar las dimensiones de la impresión.

Se han planteado distintas condiciones para evitar los cambios dimensionales, alternativas como el uso de humidificadores ${ }^{8}$, el uso de torundas de algodón ${ }^{2}$, exponerla al ambiente el menor tiempo posible, conservarla en empaques cerrados, no ser deterioradas, entre otras. Comparar las distintas condiciones permitiría conocer en qué medida afectan el cambio dimensional del modelo de yeso.

Es por este motivo que en la presente investigación se evaluó los cambios dimensionales de los modelos de yeso a partir de impresiones con hidrocoloide irreversible según distintas condiciones de tiempo de vaciado y condiciones de almacenaje en un modelo in vitro.

\section{Métodos}

Se diseñó un estudio analítico, experimental in vitro que incluyó un modelo maestro prefabricado en el cual se registraron las impresiones con hidrocoloide irreversible.

El modelo maestro consistió en cilindros de plástico confeccionados sobre una base de madera lisa y plana, los cilindros poseían $12,15 \mathrm{~mm}$ de altura y $17,25 \mathrm{~mm}$ de diámetro en su parte superior. En base al modelo se registraron 130 impresiones cuyos vaciados constituyeron las unidades de análisis (Figura 1).

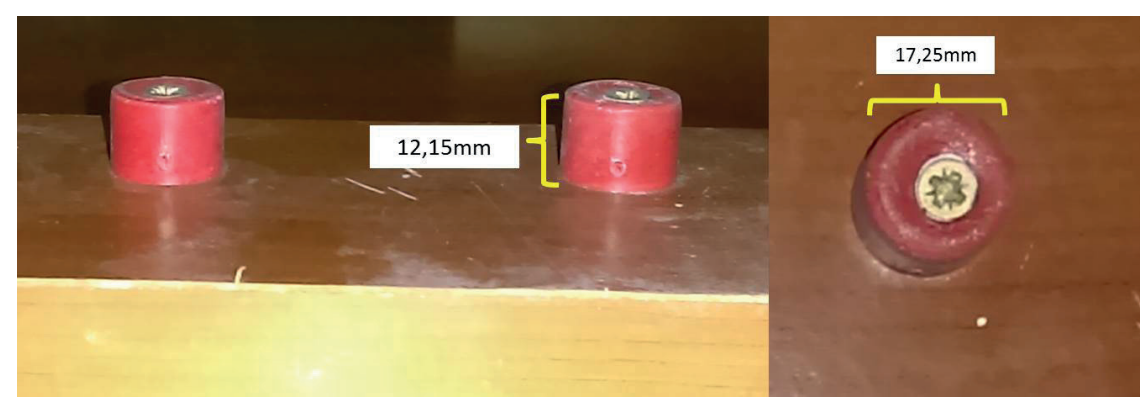

Figura 1. Dimensiones en altura y diámetro del modelo maestro 
Las impresiones se realizaron con hidrocoloide irreversible, Alginato Cromático Tropicalgin (Zhermack ${ }^{\circledR}$, Italia) según las indicaciones de la ANSI/ADA, especificación No. 18 para los materiales de impresión tipo alginato ${ }^{11}$. Se utilizaron cubetas metálicas perforadas y personalizadas de $1,5 \mathrm{~cm}$ y $2,5 \mathrm{~cm}$ de base llenándolas con más de la mitad de alginato preparado; no se utilizó algún tipo de adhesivo según las indicaciones del fabricante. La cubeta luego fue colocada encima del modelo maestro y forzándola hasta que exude el excedente de alginato preparado.

El alginato fue recolectado con una cucharada dosificadora de polvo $(9 \mathrm{~g})$ más un tercio de medidor de agua $(18 \mathrm{ml})$, la mezcla manual se realizó por $35 \mathrm{~s}$ hasta iniciar el cambio de color, tiempo en el cual se procedió a posicionar el alginato dentro de la cubeta para la posterior impresión del modelo maestro. La remoción de la cubeta se realizó de forma rápida en sentido vertical y perpendicular al modelo maestro. Obtenidas las impresiones fueron desinfectadas con spray de hipoclorito de sodio $1 \%$ sobre las superficies de la impresión por un total de $10 \mathrm{~s}$; se utilizaron tres atomizaciones para luego ser lavadas con agua corriente por $5 \mathrm{~s}$; el secado se realizó por $10 \mathrm{~s}$ a través de la jeringa de aire.

Las impresiones fueron asignadas aleatoriamente en alguno de los 13 grupos experimentales (Tabla 1).

Tabla 1. Condiciones del tiempo y almacenaje de los grupos estudiados

\begin{tabular}{|c|c|}
\hline Grupo & Característica \\
\hline Control (C) & $\begin{array}{l}\text { La impresión fue desinfectada con hipoclorito de } \\
\text { sodio } 1 \% \text { y el vaciado fue inmediato. }\end{array}$ \\
\hline Grupo 5M & $\begin{array}{l}\text { El vaciado fue luego de cinco minutos del registro } \\
\text { de impresión. }\end{array}$ \\
\hline Grupo 10M & $\begin{array}{l}\text { El vaciado fue luego de diez minutos del registro de } \\
\text { impresión. }\end{array}$ \\
\hline Grupo 15M & $\begin{array}{l}\text { El vaciado fue luego de quince minutos del registro } \\
\text { de impresión. }\end{array}$ \\
\hline Grupo 5EM & $\begin{array}{l}\text { La impresión fue almacenada en un empaque her- } \\
\text { mético y vaciado luego de cinco minutos. }\end{array}$ \\
\hline Grupo 10EM & $\begin{array}{l}\text { La impresión fue almacenada en un empaque } \\
\text { hermético y vaciado luego de diez minutos. }\end{array}$ \\
\hline Grupo 15EM & $\begin{array}{l}\text { La impresión fue almacenada en un empaque her- } \\
\text { mético y vaciado luego de quince minutos. }\end{array}$ \\
\hline Grupo 5TM & $\begin{array}{l}\text { La impresión fue llenada con torundas de algodón } \\
\text { y el vaciado luego de cinco minutos. }\end{array}$ \\
\hline Grupo 10TM & $\begin{array}{l}\text { La impresión fue llenada con torundas de algodón } \\
\text { y el vaciado luego de diez minutos. }\end{array}$ \\
\hline Grupo 15TM & $\begin{array}{l}\text { La impresión fue llenada con torundas de algodón } \\
\text { y el vaciado luego de quince minutos. }\end{array}$ \\
\hline Grupo 5ETM & $\begin{array}{l}\text { La impresión fue llenada con torundas de algodón, } \\
\text { almacenada en un empaque hermético y el vaciado } \\
\text { luego de cinco minutos. }\end{array}$ \\
\hline Grupo 10ETM & $\begin{array}{l}\text { La impresión fue llenada con torundas de algodón, } \\
\text { almacenada en un empaque hermético y el vaciado } \\
\text { luego de diez minutos. }\end{array}$ \\
\hline Grupo 15ETM & $\begin{array}{l}\text { La impresión fue llenada con torundas de algodón, } \\
\text { almacenada en un empaque hermético y el vaciado } \\
\text { luego de quince minutos. }\end{array}$ \\
\hline
\end{tabular}

Los empaques herméticos utilizados fueron de poliéster (de la marca Steriking ${ }^{\oplus}$, Finlandia) con un largo de 15 $\mathrm{cm}$ y un ancho de $5 \mathrm{~cm}$ y que son principalmente indicados para el sellado de materiales para esterilización. Las torundas de algodón fueron colocadas secas del tamaño prefabricado (Protec ${ }^{\oplus}$, México) en cada impresión se colocó cuatro torundas de algodón hasta cubrir el tope de la impresión.

Las impresiones se realizaron a una temperatura de 23 ${ }^{\circ} \mathrm{C}$ y una humedad promedio de la zona del $80 \%$; los tiempos de trabajo, manipulaciones y de fraguado se mantuvieron acorde a las indicaciones de los fabricantes. Para todos los casos del presente estudio, el lapso de tiempo que transcurrió desde el inicio de la mezcla hasta la colocación de cada una de las cubetas sobre el modelo maestro fue de 90 segundos. El vaciado de las impresiones se realizó con yeso tipo 3 (Whip Mix ${ }^{\circledR}$, EE.UU.) con mezclado manual y siguiendo las instrucciones del fabricante (proporción 1/1). Luego de una hora de fraguado se obtuvo los registros en yeso. Inmediatamente al fraguado se procedió a la inspección de cada uno de ellos mediante lentes de magnificación x2,7 (StarVision SV1 ${ }^{\oplus}$, Alemania). La existencia de poros en la superficie del yeso que impidió las mediciones excluyó al modelo. En los modelos de yeso se evaluó por un lado su diámetro y por el otro su altura en milímetros. La medición se realizó con un calibrador electrónico de precisión Vernier $\left(\right.$ Mitutoyo $^{\circledR}$, EE.UU.). Las mediciones fueron comparadas con las medidas del modelo maestro, las diferencias reflejaron los cambios dimensionales de las impresiones con alginato. Los registros lo realizaron dos investigadores (CR y YC) con una concordancia interobservador de 0,89 (Coeficiente de Correlación Intraclase) para la variable diámetro del modelo maestro.

Para el análisis de descriptivo de datos se utilizó medidas de tendencia central (media y mediana) y medidas de dispersión (desviación estándar). La prueba de Kolgomorov-Smirnov evidenció que los datos no poseían una distribución normal $(p=0,01)$ por lo que el análisis inferencial se realizó a través de pruebas no paramétricas para las medidas de altura, diámetro y diferencias con el modelo maestro. Los datos recolectados fueron tabulados en el programa MS Excel 2003 (Microsoft Corporation, EE.UU.) y analizados con el paquete estadístico SPSS v.21 (IBM ${ }^{\oplus}$, EE.UU.). La prueba de Kruskall-Wallis fue utilizada para la comparación entre los distintos grupos experimentales respecto al cambio dimensional. Una vez identificada la diferencia significativa se utilizó la prueba U-Mann Withney para la comparación entre parejas. Se aceptó un nivel de significancia de 0,05 para la refutación de la hipótesis nula.

\section{Resultados}

Al evaluar la altura de los modelos se encontró que el Grupo 15ETM (impresión llenada con torundas de algodón, almacenada en un empaque hermético y vaciada luego de quince minutos) presentó la medida $(12,45 \pm 0,22 \mathrm{~mm})$ más cercana al patrón origi- 
nal $(12,15 \mathrm{~mm})$ mientras que el Grupo $5 \mathrm{M}$ (vaciado luego de cinco minutos del registro de impresión) presentó la medida $(13,12 \pm 0,2 \mathrm{~mm})$ más lejana al patrón original. Se encontró diferencias significativas en los grupos 5TM $(p=0,01) 10 \mathrm{TM}(p=0,02), 15 \mathrm{TM}$ $(p=0,01)$ y $5 \operatorname{ETM}(p=0,03)$ respecto al modelo maestro (Tabla 2).

El diámetro del modelo de yeso del Grupo 5TM (uso de torunda y vaciado en cinco minutos) fue el más cercano al modelo patrón con una media de 17,3 \pm 0,03 $\mathrm{mm}$; mientras que el diámetro del Grupo $10 \mathrm{M}$ (vaciado luego de diez minutos) evidenció la medida más diferente con una media de 17,48 $\pm 0,14 \mathrm{~mm}$. Se encontraron diferencias significativas a nivel de los grupos $5 \mathrm{M}(p=0,02), 15 \mathrm{M}(p=0,01), 5 \mathrm{EM}(p=0,01), 10 \mathrm{EM}$ $(p=0,03)$, y $15 \mathrm{EM}(p=0,02)$, respecto al modelo maestro (Tabla 2).

Tabla 2. Dimensiones en altura y diámetro de los modelos de yeso obtenidos por grupo de estudio

\begin{tabular}{lcc}
\hline Grupo experimental & Altura $(\mathrm{mm})$ & Diámetro (mm) \\
Grupo C & $12,56 \pm 0,38$ & $17,31 \pm 0,04$ \\
Grupo 5M & $13,12 \pm 0,2^{*}$ & $17,4 \pm 0,09^{*}$ \\
Grupo 10M & $13,04 \pm 0,23$ & $17,48 \pm 0,1^{*}$ \\
Grupo 15M & $13,05 \pm 0,23$ & $17,45 \pm 0,09^{*}$ \\
Grupo 5EM & $12,93 \pm 0,33$ & $17,35 \pm 0,07^{*}$ \\
Grupo 10EM & $12,89 \pm 0,22$ & $17,46 \pm 0,16^{*}$ \\
Grupo 15EM & $13,02 \pm 0,22$ & $17,44 \pm 0,18^{*}$ \\
Grupo 5TM & $12,53 \pm 0,28^{*}$ & $17,3 \pm 0,03^{*}$ \\
Grupo 10TM & $12,48 \pm 0,17^{*}$ & $17,38 \pm 0,07$ \\
Grupo 15TM & $12,46 \pm 0,22^{*}$ & $17,41 \pm 0,01$ \\
Grupo 5ETM & $12,73 \pm 0,36^{*}$ & $17,37 \pm 0,05^{*}$ \\
Grupo 10ETM & $12,72 \pm 0,3$ & $17,45 \pm 0,14$ \\
Grupo 15ETM & $12,45 \pm 0,22^{*}$ & $17,33 \pm 0,12$ \\
Total & $12,76 \pm 0,35$ & $17,39 \pm 0,12$ \\
Patrón & 12,15 & 17,25 \\
\hline
\end{tabular}

\section{* $\mathrm{H}$ Kruskal-Wallis $p<0,05$.}

Grupo C (Vaciado inmediato). Grupo 5M (Vaciado en $5 \mathrm{~min}$ ). Grupo $10 \mathrm{M}$ (Vaciado en $10 \mathrm{~min}$ ). Grupo 15M (Vaciado en $15 \mathrm{~min}$ ). Grupo 5EM (Empaque y vaciado en $5 \mathrm{~min}$ ). Grupo 10EM (Empaque y vaciado en 10 $\mathrm{min})$. Grupo 15EM (Empaque y vaciado en $15 \mathrm{~min}$ ). Grupo 5TM (Torunda y vaciado en $5 \mathrm{~min}$ ). Grupo 10TM (Torunda y vaciado en $10 \mathrm{~min}$ ). Grupo 15TM (Torunda y vaciado en $15 \mathrm{~min}$ ). Grupo 5ETM (Torunda, empaque y vaciado en $5 \mathrm{~min}$ ). Grupo 10ETM (Torunda, empaque y vaciado en 10 $\mathrm{min}$ ). Grupo 15ETM (Torunda, empaque y vaciado en $15 \mathrm{~min}$ ).

El menor cambio dimensional respecto a la altura se encontró con el Grupo 15ETM con una media de 0,3 \pm $0,22 \mathrm{~mm}(p=0,01)$ (Tabla 1$)$, mientras que a nivel del diámetro el Grupo C $(0,06 \pm 0,04 \mathrm{~mm})$ y el Grupo
5TM $(0,05 \pm 0,03 \mathrm{~mm})$ evidenciaron los menores cambios dimensionales $(p=0,01)$ (Tabla 3$)$.

Tabla 3. Cambios dimensionales de los modelos de yeso respecto al modelo maestro

\begin{tabular}{|c|c|c|}
\hline $\begin{array}{c}\text { Grupo } \\
\text { experimental }\end{array}$ & $\begin{array}{l}\text { Diferencia con la } \\
\text { altura patrón }\end{array}$ & $\begin{array}{l}\text { Diferencia con el } \\
\text { diámetro patrón }\end{array}$ \\
\hline Grupo C & $0,41 \pm 0,38$ & $0,06 \pm 0,04^{*}$ \\
\hline Grupo 5M & $0,97 \pm 0,2$ & $0,15 \pm 0,09$ \\
\hline Grupo 10M & $0,89 \pm 0,23$ & $0,23 \pm 0,14$ \\
\hline Grupo 15M & $0,9 \pm 0,23$ & $0,2 \pm 0,09$ \\
\hline Grupo 5EM & $0,78 \pm 0,33$ & $0,1 \pm 0,07$ \\
\hline Grupo 10EM & $0,74 \pm 0,22$ & $0,21 \pm 0,16$ \\
\hline Grupo 15EM & $0,87 \pm 0,22$ & $0,19 \pm 0,18$ \\
\hline Grupo 5TM & $0,38 \pm 0,28$ & $0,05 \pm 0,03^{*}$ \\
\hline Grupo 10TM & $0,33 \pm 0,17$ & $0,13 \pm 0,07$ \\
\hline Grupo 15TM & $0,31 \pm 0,22$ & $0,16 \pm 0,01$ \\
\hline Grupo 5ETM & $0,58 \pm 0,36$ & $0,12 \pm 0,05$ \\
\hline Grupo 10ETM & $0,57 \pm 0,3$ & $0,2 \pm 0,14$ \\
\hline Grupo 15ETM & $0,3 \pm 0,22^{*}$ & $0,08 \pm 0,12$ \\
\hline Total & $0,61 \pm 0,35$ & $0,14 \pm 0,12$ \\
\hline Patrón & 0,00 & 0,00 \\
\hline $\begin{array}{l}{ }^{*} \\
\text { Grupo C (Vaciado } \\
10 \mathrm{M} \text { (Vaciado en } 10 \\
\text { (Empaque y vaciadd } \\
\text { min). Grupo } 15 \mathrm{EM}( \\
\text { y vaciado en } 5 \text { min). } \\
\text { 15TM (Torunda y va } \\
\text { y vaciado en } 5 \text { min) } \\
\text { min). Grupo } 15\end{array}$ & $\begin{array}{l}\text { hoc de Kruskal-Wallis } \\
\text { diato). Grupo } 5 \mathrm{M} \text { (Va } \\
\text {. Grupo } 15 \mathrm{M} \text { (Vaciad } \\
\text { min). Grupo } 10 \mathrm{EM} \text { ( } \\
\text { que y vaciado en } 15 \mathrm{n} \\
\text { o } 10 \mathrm{TM} \text { (Torunda y v } \\
\text { en } 15 \text { min). Grupo } 5 \\
\text { oo } 10 \mathrm{ETM} \text { (Torunda, } \\
\text { (Torunda, empaque y }\end{array}$ & $\begin{array}{l}, 05 \\
\text { do en } 5 \text { min). Grupo } \\
15 \text { min). Grupo } 5 \text { EM } \\
\text { daque y vaciado en } 10 \\
\text {. Grupo } 5 T M \text { (Torunda } \\
\text { do en } 10 \text { min). Grupo } \\
\text { (Torunda, empaque } \\
\text { daque y vaciado en } 10 \\
\text { ciado en } 15 \text { min). }\end{array}$ \\
\hline
\end{tabular}

\section{Discusión}

Los hidrocoloides irreversibles modifican su estado físico de sólido a gel, cuando su volumen de agua disminuye la impresión se contraerá y si aumenta se expandirá. Los geles pierden volumen de agua por evaporación y/o por el fenómeno de sinéresis, por el contrario pueden ganarla por el fenómeno de imbibición. Los cambios dimensionales de los hidrocoloides (alginatos) pueden modificar los registros que se toman y alterar la precisión y exactitud de las características de los registros en positivo (modelos de yeso).

De los distintos factores que influyen en el cambio de la estabilidad dimensional de los modelos de yeso bajo las impresiones con alginato se han mencionado: tiempo de vaciado, grado de humedad, condiciones de almacenamiento, características de la preparación/mezclado y marca del material. Nuestro estudio encontró que los grupos donde se utilizó torundas de algodón y empaques herméticos evidenciaron menores cambios dimensionales, siendo significativos. Quizás puede entenderse este fenómeno debido a que el grado de humedad en la que se encuentra almacenada la impresión influye en sus 
posteriores dimensiones, esto debido a que la disminución de la humedad puede contraer al alginato. Se recomienda que las impresiones deban almacenarse con un $100 \%$ de humedad lo cual es conseguido utilizando recipientes y/o envases con cierre hermético ${ }^{12,13}$, se ha sugerido el uso de paños húmedos al lado de la impresión que permitan aumentar el grado de humedad. Kulami y cols. ${ }^{14}$, plantean que las condiciones de almacenamiento reducen los cambios dimensionales en las primeras dos horas de vaciado, asimismo Ismail y cols. ${ }^{15}$ acotan que el alginato almacenado en humedad relativa del $100 \%$, después de 24 horas posee una exactitud parecida a la de los elastómeros y finalmente Khaledi y cols. ${ }^{16}$ concluyen que los hidrocoloides estudiados se pueden almacenar en un ambiente húmedo durante tres horas, sin sufrir cambios significativos. Nuestro estudio confirmó que el agregar una torunda o empaques disminuye el cambio dimensional respecto a la altura y diámetro cuando se comparó con no utilizar algún elemento que proteja de las condiciones ambientales.

Referidos al tiempo de vaciado algunos estudios indican que las modificaciones dimensionales ocurren desde el primer minuto de la impresión ${ }^{17}$ debido a que existen cambios ambientales como humedad y temperatura en el lugar de trabajo ${ }^{18}$; sin embargo, algunos estudios al cabo de diez minutos de vaciado no encontraron diferencias significativas ${ }^{19}$. Nuestro estudio encontró que el vaciado inmediato evidenció cambios dimensionales similares con los grupos de vaciado a los cinco minutos si se utiliza torundas y/o torunda más un empaque hermético. Asimismo referidos al cambio en la altura de las impresiones se encontró que los mayores cambios dimensionales fueron con los vaciados luego de 5,10 y 15 minutos, así como al utilizar empaques herméticos. Shaba y cols. ${ }^{14}$ indican que el vaciado debe ser realizado dentro de los diez minutos de realizada la impresión para obtener los menores cambios dimensionales con el alginato. A diferencia de lo indicado en la literatura nuestro estudio encontró que el utilizar torunda, empaque y vaciado luego de 15 minutos no evidenció mayor cambio dimensional sino que por el contrario se encontró el mínimo cambio dimensional.

También se ha encontrado que según los tipos de marcas es modificable la estabilidad dimensional de los alginatos $1,9,10$, algunos estudios indican que los más estables son el Alginoplast y el Hydrogun ${ }^{9}$. Los alginatos cromáticos han demostrado no presentar diferencias significativas respecto a un modelo maestro de metal en cortos periodos de vaciado ${ }^{20,21}$. De manera general nuestro estudio corrobora esta información, ya que en todos los grupos estudiados y el alginato cromático utilizado evidenció cambios dimensionales pequeños que oscilaron entre 0,06-0,97 mm; esto señala que antes de los 15 minutos de vaciados los cambios dimensionales son mínimos, pero no por eso se debe prolongar el tiempo de vaciado. Se ha aconsejado que las impresiones deben ser vaciadas antes de los 30 minutos porque existen cambios dimensionales que obligarán a repetir la impresión con alginato ${ }^{22}$. En nuestra investigación el alginato uti- lizado no evidenció amplios cambios dimensionales al cabo de 15 minutos de vaciado, pese a que otros autores como Iwasaki y cols. ${ }^{23}$ sugieren que las impresiones con Tropicalgin (Zhermack) deben ser vaciadas inmediatamente, o lo más rápido posible dentro de las primeras 24 horas, así también Todd y cols. ${ }^{6}$ concluyen que la mayor cantidad de cambios dimensionales se generan en las primeras 24 horas, lo que indica que a mayor tiempo transcurrido desde la toma de impresión se genera mayores cambios dimensionales.

Nuestro estudio posee las limitaciones del potencial error atribuible al instrumento de medición al momento de realizar las mediciones, así como la contracción del material de impresión por otros factores que no pudieron ser controlados, tales como el porcentaje de humedad o las alteraciones en la superposición.

Los grupos que fueron almacenados con torundas y empaques herméticos evidenciaron los menores cambios dimensionales a los 5, 10 y 15 minutos. Por lo cual, concluimos que aun siendo los cambios dimensionales con el hidrocoloide irreversible muy pequeños al cabo de 15 minutos de vaciado, estas modificaciones son menores cuando las impresiones son almacenadas en empaques herméticos y con el uso de torundas de algodón que rellenen la impresión.

\section{Referencias bibliográficas}

1. Bayindir F, Yanikoglu N, Duymus Z. Thermal and $\mathrm{pH}$ changes, and dimensional stability in irreversible hydrocolloid impression material during setting. Dent Mater J. 2002;21(2):200-09.

2. Rodrigues SB, Augusto CR, Leitune VC, Samuel SM, Collares FM. Influence of delayed pouring on irreversible hydrocolloid properties. Braz Oral Res. 2012;26(5):4049. doi.org/10.1590/S1806-83242012000500005.

3. Fellows CM, Thomas GA. Determination of bound and unbound water in dental alginate irreversible hydrocolloid by nuclear magnetic resonance spectroscopy. Dent Mater. 2009;25(4):486-93. doi: 10.1016/j.dental.2008.10.001.

4. Macchi RL. Materiales Dentales. Buenos Aires: Médica Panamericana; 1980.185 p.

5. Cova N. Biomateriales dentales. Caracas: Amolca; 2010.

6. Todd JA, Oesterle LJ, Newman SM, Shellhart WC. Dimensional changes of extended-pour alginate impression materials. Am J Orthod Dentofacial Orthop. 2013;143(4 Suppl):S55-63. doi: 10.1016/j.ajodo.2012.06.016.

7. Alvarez A. Estudio comparativo de la estabilidad dimensional del alginato Kromopan 100 hours (Lascod) contra Tropicalgin (Zhermack). [Tesis de Licenciatura]. Universidad Nacional Autónoma de México. 2009.

8. Anusavice KJ. Phillips ciencia de los materiales dentales. 11a ed. Madrid: Elsevier; 2004.

9. Sedda M, Casarotto A, Raustia A, Borrachín A. Effect of storage time on the accuracy of casts made from different irreversible hidrocolloid. J Contemp Dent Pract. 2008;9(4):58-66. 
10. Cohen BI, Pagnillo M, Deutsch AS, Musikant BL. Dimensional accuracy of three different alginate impression materials. J Prosthodont. 1995;4(3):195-9.

11. ANSI/ADA AN. Specification No. 18 for Alginate Impression Material. American National Standarts Institute. 1992.

12. Bernard S, Paul W. Utilización clínica de los materiales dentales. Barcelona: Ed Masson. 1996.

13. Kulkarni MM, Thombare RU. Dimensional Changes of Alginate Dental Impression Materials-An Invitro Study. J Clin Diagn Res. 2015;9(8):ZC98-ZC102. doi: 10.7860/ JCDR/2015/13627.6407

14. Shaba OP, Adegbulugbe IC, Oderinu OH. Dimensional stability of alginate impression material over a four hours' time frame. Nig Q J Hosp Med. 2007;17(1):1-4.

15. Ismail HA, Mahross HZ, Shikho S. Evaluation of dimensional accuracy for different complete edentulous impressions immersed in different disinfectant solutions. Eur J Dent. 2017;11(2):242-9. doi: 10.4103/ejd. ejd_268_16.

16. Khaledi A, Borhanihaghighi Z, Vojdani M. The effect of disinfectant agents on dimensional stability and surface roughness of a tissue conditioner material. Indian J Dent Res. 2011;22(4):499-504. doi: 10.4103/09709290.90277.

17. Fellows CM, Thomas GA. Determination of bound and unbound water in dental alginate irreversible hydrocolloid by nuclear magnetic resonance spectroscopy. Dent Mater. 2009;25(4):486-93.

18. Sawyer HF, Sandrik JL, Neiman R. Accuracy of casts produced from alginate and hidrocolloid impression materials. J Am Dent Assoc. 1976;93(4):806-8.

19. Nallamuthu NA, Braden M, Patel MP. Some aspects of the formulation of alginate dental impression materials--setting characteristics and mechanical properties. Dent Mater. 2012;28(7):756-62. doi: 10.1016/j.dental.2012.03.012.

20. Ibrahim AA, Alhajj MN, Khalifa N, Gilada MW. Does 6 Hours of Contact With Alginate Impression Material Affect Dental Cast Properties? Compend Contin Educ Dent. 2017;38(6):e1-e4.

21. Muzaffar D, Ahsan SH, Afaq A. Dimensional changes in alginate impression during immersion in a disinfectant solution. J Pak Med Assoc. 2011;61(8):756-9.

22. Inoue $S$, Kawara M, Iida T, Iwasaki M, Komiyama O. Influences of differences in tray design and impression material on impression pressure at edentulous mandible. J Oral Sci. 2017;59(4):505-10. doi: 10.2334/josnusd.16-0731. [Epub ahead of print]

23. Iwasaki Y, Hiraguchi H, Iwasaki E, Yoneyama T. Effects of immersion disinfection of agar-alginate combined impressions on the surface properties of stone casts. Dent Mater J. 2016;35(1):45-50. doi: 10.4012/dmj.2015-163. 